\title{
Die Nutzung audiovisueller Medien durch Kinder und Jugendliche
}

Summary: Audiovisual media dominate the leisure time of children and adolescents. Television is still the main medium. This dominant use of television is illustrated by data representative for Germany. Differences in television use by age, sex, social background are demonstrated and partly explained by ideas, stemming from the uses and the gratification approach. The media related behaviour of parents is also discussed. Secular trends, caused by the introduction of private television, are pointed out. Finally, the connections between television use and reading (which seems to be a displacement relation) and between television and video (supplement relation) are demonstrated.

\section{Mediennutzungen und nicht-mediale Freizeittätigkeiten}

Medien bilden einen Teil der inner- und außerhäuslichen Ökologie, sie eröffnen Nutzungsmöglichkeiten und sie verlocken zu ihrem Gebrauch. Dabei versuchen die Medienproduzenten, durch hohe Attraktivität Kinder und Jugendliche als Konsumenten zu Kauf oder Nutzung zu gewinnen. Auf der Medienseite sind hierbei sowohl die Möglichkeiten, die sich durch technologische Neuerungen (hardware) eröffnen, zu berücksichtigen, wie auch die auf dieser Basis entwickelten Angebote und Angebotsformen (software).

Attraktivität ist aber immer ein Wechselwirkungsprodukt aus der Übereinstimmung von Bedürfnis- und Angebotsaspekten. Es ist also von einer bedürfnisgesteuerten Selektion aus den medialen Angeboten auszugehen, wobei mit diesem Terminus nicht impliziert ist, daß die Zuwendung zu den Medien immer bewußt oder ausschließlich rational gesteuert stattfindet. Auf der Nutzerseite ist für die Medienzuwendung die verfügbare Freizeit wesentlich, dann sind eingeschliffene Verhaltensgewohnheiten, durch die der Alltag strukturiert wird, zu berücksichtigen. Bedürfnisse der Kinder und Jugendlichen i.e.S. sind für die Medienzuwendungen aber ebenso wichtig, wobei neben den aus der Mediennutzung erwartbaren Gratifikationen auch die erreichbaren Freizeitalternativen und Freizeitkompetenzen eine wesentliche Rolle spielen. Schließlich haben auch Eltern Vorstellungen über angemessenen Medienkonsum und versuchen, ihre Kinder in ihrem Sinn zu beeinflussen. 
Nicht zu vergessen, Mediennutzungen sind in soziale Bezüge eingebunden. Dabei ist sowohl an die Möglichkeit zu denken, daß Mediennutzungen eine Kompensation für nicht vorhandene Sozialkontakte sein können, zum anderen aber eine soziale Aktivität darstellen, durch die sich Status und Prestige in den jeweiligen Peer-Gruppen erhöhen lassen. Auch hierbei ist zu vermuten, daß jedes Medium eine jeweils unterschiedliche soziale Valenz besitzt.

$\mathrm{Da}$ Medien einen hohen zeitlichen Stellenwert im Rahmen aller Freizeittätigkeiten einnehmen, ist seit einer Tagesablaufstudie von BESSLER und ZIMMERSCHÜRINGS (1973) bekannt. Besonders wird in diesen Untersuchungen die Konkurrenz zwischen dem freien Spielen der Kinder und dem Ausmaß ihrer Medienzuwendungen deutlich: Im Vorschulalter ist noch eine beträchtliche Dominanz des Spielens vorhanden (täglich 284 min./Medientätigkeiten 66 min.), die im Grundschulalter stark zurückgeht (174 min. Spiel/Medientätigkeiten 113 min.); in der nächsthöheren Altersgruppe nehmen die Medientätigkeiten (160 min.) mehr Zeit als das Spiel (115 min.) ein. Bereits bei dieser frühen Erhebung hat von den Medientätigkeiten das Femsehen den größten Zeitanteil $(67,5 \%)$ ausgemacht - ein Befund, der über alle späteren Untersuchungen stabil geblieben ist. Nachweislich nimmt das Femsehen zwischen dem 6. und dem 13. Lebensjahr einen von 19 auf $24 \%$ steigenden Anteil der Freizeit ein (Infratest, 1981, S. 327).

Um den säkularen Trend deutlich zu machen, kann auf die neuere Studie von TIETZE (1990, S. 267) verwiesen werden. Danach haben im Vorschulalter (3- bis 6jährige) die gezielten Medienzuwendungen zugenommen - sie machen insgesamt täglich 85 Minuten aus. Rechnet man noch die Zeit hinzu, während der Kinder Medien im Sinne einer Nebentätigkeit nutzen, so erhöht sich dies auf durchschnittlich 146 Minuten. Nur 4\% der Kinder hatten an dem Erhebungsstichtag keine Medien verwendet. Auch aus der „Kinder und Medien Studie 1990“ (KLINGLER \& GROEBEL, 1994) ist schließlich ersichtlich, daß die Spielzeiten während der Kindheit ( 6 bis 13 Jahre) zurückgehen und die medienbezogenen Tätigkeiten zunehmen. Nach dieser Studie übertreffen aber die medienbezogenen Tätigkeiten das Spiel in quantitativer Hinsicht nicht.

\section{Das Fernsehen als audiovisuelles Leitmedium}

\subsection{Haushaltsausstattungen mit Fernsehgeräten}

Trotz aller technischer Neuentwicklungen ist das Fernsehen immer noch als das audiovisuelle Leitmedium anzusehen. Dies hängt mit seiner ubiquitären Verfügbarkeit zusammen, denn ein Femsehapparat ist seit langem in ca. $98 \%$ aller Haus- halte vorhanden. Der Femsehapparat wurde auch als modemer „Hausaltar“ bezeichnet, nimmt er doch in den meisten Fällen eine dominante Position im Wohnzimmer ein (nach SAXER et al. [1989, S. 40] ist er in $89 \%$ der Fälle im Wohnzimmer aufgestellt, die Zweitgeräte werden hingegen am häufigsten im Kinderzimmer oder im Schlafzimmer plaziert). Auch die Satellitenantenne oder ein Kabelanschluß sind zwischenzeitlich in drei Viertel der Haushalte verfügbar (MA '94: Westhaushalte mit einem Jugendlichen 73\%, Osthaushalte 79\%).

In etwa einem Viertel der Haushalte mit Kindem ist ein Zweitgerät vorhanden (TieTZE, 1990, S. 268). Nach der „Kinder und Medien Studie 1990“ (KLINGLeR \& GroEBEL, 1994) besitzen $19 \%$ aller 6- bis 13jährigen ein eigenes Gerät (und zwar $17 \%$ der West- und 25\% der Ost-Kinder). Durch ein Zweitgerät in den Familien verringert sich die potentielle Konfliktmöglichkeit wegen des auszuwählenden Programms, zugleich geht auch der Einfluß der Eltem auf die Sehinhalte ihrer Kinder entsprechend zurück.

\subsection{Alterstrends bezüglich des Femsehens}

Wann beginnen Kinder femzusehen? Sieht man einmal von Befunden zum sog. Babyfemsehen ab (KLINGLER \& Groebel, 1994, S. 213), das in Ballungsgebieten Japans aufgrund beengter Wohnverhältnisse zu den menschlichen „Früherfahrungen“" gehört, so scheint sich ab dem 2. Lebensjahr eine mehr oder minder regelmäßige Femsehzuwendung abzuzeichnen: Zumindest nach Mütterangaben weisen von den dreijährigen Kindem $57 \%$ eine einjährige und $29 \%$ eine halbjährige Seherfahrung auf (FIRNKES et al., 1973, S. 22).

Im 2. Lebensjahr ist die Qualität des Fernsehens durch kurzfristige Zuwendungen, hohe Ablenkbarkeit und im Vergleich zu älteren Kindem durch eine andersgeartete Informationsentnahme gekennzeichnet. Ab dem 3. Lebensjahr ist eine gezielte Femsehzuwendung zu beobachten. Ab diesem Alter haben sich feste Sehzeiten und Spartenkenntnisse (z.B. Werbung) etabliert.

Nach TiETZE (1990) betragen die Sehzeiten für Vorschulkinder 36 Minuten; nach einer Analyse der GfK-Daten von 1995 sind für drei- bis fünfjährige Kinder zwischenzeitlich Sehzeiten von 74 Minuten typisch (FEIERABEND \& WINDGASSE, 1996, S. 186). Bei Kindem im Grundschulalter ist ein Anstieg der Sehzeit festzustellen ( 92 Minuten bei 6- bis 9jährigen), der sich kontinuierlich bis zum Ende der Sekundarstufe I fortsetzt (10- bis 13jährige 114 Minuten, a.a.O.).

Die weitere Entwicklung ist durch einen Rückgang bei den Präadoleszenten (16- bis 19jährige) und einen Anstieg bei den Postadoleszenten (25- bis 29jährige) gekennzeichnet (BoNFADELlı et al., 1986, S. 143). Dieses Veränderungsmuster ist 
international gut dokumentiert (für die Schweiz vgl. Aregger \& Steinmann [1989, S. 92], für Schweden vgl. Rosengren \& Windahl [1989, S. 21 f.]).

Von der durchschnittlichen Sehzeit zu unterscheiden sind Reichweiteangaben (damit ist gemeint, welcher Anteil einer bestimmten Altersklasse pro Tag mit dem Medium in Kontakt kommt). Bei Vorschulkindern beträgt die Reichweite des Fernsehens während der Woche 58\%, ein Drittel der Kinder sieht täglich, knapp 15\% schauen unter der Woche gar nicht fern (TIETZE, 1990, S. 268). Dies bedeutet auch, daß die Durchschnittszeiten bei den tatsächlichen Sehern entsprechend höher liegen.

Nach der „Kinder und Medien Studie 1990“ ist das Fernsehen (außer der Lerntätigkeit für die Schule) die am häufigsten ausgeführte Freizeitaktivität von Kindern (KLINGLER \& GROEBEL, 1994, S. 55): Von $77 \%$ aller 6- bis 13jährigen in den alten Bundesländern wird täglich ferngesehen, von weiteren $17 \%$ zumindest wöchentlich. Eine Zunahme mit dem Alter läßt sich nachweisen (6- und 7jährige ca. $67 \%$ täglich, 12- und 13jährige ca. $81 \%$ ). In den neuen Bundesländern übertrifft die Häufigkeit des Fernsehens (täglich $93 \%, 4 \%$ wenigstens wöchentlich) sogar die Häufigkeit des Lernens für die Schule (täglich 90\%).

$\mathrm{Da}$ das Fernsehen in unterschiedlichen Gruppen anders genutzt wird, ist lange bekannt. Sozialschichtunterschiede deuten international übereinstimmend darauf hin, daß untere soziale Gruppen länger fernsehen (FILIPSON, 1978, S. 67; ROSENGREN \& WINDAHL, 1989; FURU, 1971). Ebenso zeigen Jungen eine stärkere Bindung an das Fernsehen als Mädchen (LUKESCH et al., 1994; FURU, 1971). Dies bezieht sich sowohl auf Sehzeiten wie auf Präferenzurteile (FILIPSON, 1978, S. 67; AREGGER \& STEINMANN, 1989, S. 97) und hat sich schon in früheren Lebensjahren gefestigt (ROSENGREN \& WiNDAHL, 1989, S. 21 ff.).

Das Fernsehnutzungsmuster von Kindern ist (ebenso wie bei Erwachsenen) durch die Möglichkeit des Umschaltens (Zappen) und des Scannens durch die Programme gekennzeichnet. Es wird also nicht kohärent eine Sendung nach der anderen angeschaut, sondern längerdauernde Zuwendungen wechseln mit kurzfristig wechselnden Einschaltungen ab (KLINGLER \& GROEBEL, 1994, S. 204). Ob damit ein „kreativer“ Umgang mit dem Fernsehen etabliert wird, ist anzuzweifeln Die Umschaltvorgänge sind eher als Versuche des Erregungsmanagements interpretierbar. In welcher Weise sich dies auf die Rezeption von Medienbotschaften (die ja nicht mehr als eigenständige Werke rezipiert werden) auswirkt, ist bislang ungeklärt.

Ebenso muß bedacht werden, daß in Europa - wie bereits seit längerer Zeit in den USA - das Fernsehen den Charakter eines „Nebenbeimediums“ erhalten hat (KROTZ, 1994, S. 505). Das kann bedeuten, daß nur mehr die Handlungselemente einer Sendung bewußt wahrgenommen werden, die eine sehr hohe Erregungswirkung haben oder die zu Voreinstellungen der Rezipienten gut passen.
2.3 Beliebtheit des Fernsehens

Bei spontanen Angaben über Lieblingstätigkeiten steht das Spielen (46\%) noch vor dem Fernsehen (34\%), dann kommen Radfahren (28\%), sportliche Betätigungen $(26 \%)$ und das Lesen (24\%) (KLINGLER \& GrOebel, 1994, S. 53). Bei strukturierten Vorgaben liegt das Fernsehen nach der Beliebtheit bei den Westkindern an dem 4. Rangplatz von 26 vorgegebenen Freizeitbetätigungen (wobei 80\% ihm eine hohe Präferenz zusprechen), nur das Zusammensein mit FreundInnen, das Spielen im Freien und Sportvereinsstunden sind noch beliebter. Bei den Ostkindern liegt nach der Beliebtheit das Fernsehens mit einer Präferenz von $91 \%$ unangefochten an der Spitze aller Freizeitbetätigungen.

Da Kinder lieber außer Haus mit Freunden ihre Zeit verbringen (AREGGER \& Steinmann, 1989, S. 87; Fil IPSON, 1978, S. 67), ist zu erwarten, daß sich bei außerhäusigen Beschränkungen der Fernsehkonsum erhöht. Dies belegt die saisonale Betrachtung der Fernsehnutzung (Media Perspektiven, 1989-1996): Im ersten und letzten Quartal eines jeden Jahres nehmen bei Kindern sowohl die Fernsehreichweiten um ca. $10 \%$ und die Sehzeiten um etwa 20 Minuten pro Tag zu. Generalisierend ist festzuhalten, die Fernsehnutzung steigt, wenn ,nichts Besseres zu tun" ist (z.B. wegen mangelnder Spielmöglichkeiten in der Wohnumgebung, Wettergegebenheiten). Aber auch andere attraktive Alternativen können den Fernsehkonsum dämpfen, so steigt die Fernsehnachfrage bei mangelnden Spielmöglichkeiten in der Wohnumgebung (SCHNEEWIND et al., 1983) oder bei Krankheit des Kindes (LUKESCH, 1993).

\subsection{Fernsehen im Tagesverlauf}

Bei Vorschulkindern liegt ein Nutzungshöhepunkt des Fernsehens zwischen 9 und $10 \mathrm{Uhr}$ vormittags (Reichweite 9\%), ein weiterer zwischen 18 und $19.30 \mathrm{Uhr}$ (Reichweite $27 \%$ ) (TIETZE, 1990, S. 270 f). Bei den 6- bis 13jährigen ist (wegen der Schule) der Fernsehgipfel auf 18 bis 21 Uhr verschoben (KLINGLER \& GROEBEL, 1994, S. 134), wobei erwartbare Unterschiede nach Wochentagen (spätere Sehzeiten speziell an Samstagen) und nach dem Alter der Kinder (kontinuierliche Verschiebung des Fernsehens auf die späteren Stunden) auftreten.

Die Sehzeiten haben sich im Zeitvergleich bedeutsam auf später verschoben (BESSLER \& ZimmER-SChÜrings, 1973, S. 243). Nach ECKARDT (1989) sind von den 6- bis 9jährigen etwa $25 \%$ nach $20 \mathrm{Uhr}$ vor dem Fernseher zu finden, von den 10- bis 13jährigen sogar mehr als $35 \%$. Nach 22 Uhr schauen nach der ARD-ZDF Studie von den 6- bis 13 jährigen 4,2\% der Westkinder bzw. 2,7\% der Ostkinder 
noch ferm; dieser Prozentsatz mag nicht als sehr hoch erscheinen, absolut gesehen sind es aber ca. 243000 West- und 52500 Ostkinder, die z.T. altersbeschränkte Programme sehen. Nach GfK-Daten (FEIERABEND \& WindGasse, 1996, S. 188) sehen zwischen 21 und 24 Uhr $6 \%$ der 3- bis 13jährigen noch ferm, in absoluten Zahlen sind dies für ganz Deutschland 550000 Kinder.

Festzuhalten ist, daß heute bei weniger Kindem die Sehzeit durch die Eltern beschränkt wird. Die gewachsene Gleichgültigkeit der Eltem gegenüber den Sehwünschen der Kinder (trotz der auf anderer Seite demonstrativ geäußerten Besorgnisse) zeigt sich auch darin, daß abendliche Beschränkungen der Sehzeiten weniger mit den Programminhalten begründet werden und mehr mit formalen Gründen (z.B. Aufstehen wegen Schule).

\subsection{Femsehnutzung und Empfangsmöglichkeiten}

Die Fermsehnutzung muß nach den Angeboten der öffentlich-rechtlichen Fernsehanstalten und der Privatsender differenziert werden. Aus den GfK-Messungen kann eine gewisse Nutzungszunahme bei zusätzlichen Empfangsmöglichkeiten abgeleitet werden (DARSCHIN \& FrANK, 1991, S. 186). Dies sei an zwei Eckdaten illustriert: Die von ECKARDT (1989) erarbeitete Gegenüberstellung der Sehdauer von Kindem aus dem GfK-Panel für 1988 zeigte, daß die durchschnittliche tägliche Sehzeit in Haushalten mit Empfangsmöglichkeit von Privatfemsehen 7 Minuten höher ist als in Haushalten ohne Privatempfang. Die von HuRRELMANN (1989, S. 48) aus dem Kabelpilotprojekt Dortmund mitgeteilten Zahlen ( $N=304$ Familien) belegen hingegen, daß in verkabelten Haushalten wesentlich höhere durchschnittliche Sehzeiten vorliegen: Bei Kindem bis 3 Jahren verdoppelt sich die Sehzeit von 17 auf 33 Minuten, bei 4- bis 6jährigen nimmt sie von 52 auf 87 Minuten und bei 7- bis 9jährigen von 81 auf 113 Minuten zu (vgl. auch TIETZE, 1990).

Selbst bei älteren Kindem zeigt sich ein moderater Einfluß des zusätzliches Fermsehangebotes. Nach einer Sonderauswertung der GfK-Daten für Jugendliche (14- bis 19jährige; van Eimeren \& KLINGLER, 1995, S. 212) sind z.B. die Reichweiten für das Fernsehen zwischen Haushalten mit Kabel-/Satellitenanschluß und ohne diesem nicht wesentlich unterschiedlich (56 bzw. $55 \%$ ), aber die Sehzeiten differieren um fast 15 Minuten (99 vs. 84 Minuten/Tag). Eltem, die sich für ein erweitertes Femsehangebot entschieden haben, schätzen das Femsehen zudem wesentlich positiver ein als Nicht- Kabelteilnehmer. Es wird aber keineswegs mehr fermsehbezogene Unterstützung gewährt (z.B. in Form gemeinsamen Sehens oder erläutemder Gespräche), sondem das Femsehen wird vermehrt zur Eltementlastung eingesetzt (HuRrElmanN, 1989, S. 17) und „Kabel-Eltem“ sehen im gestiegenen und andersartigen Femsehkonsum ihrer Kinder auch keine besondere pädagogische Herausforderung (HuRRELMANN, 1989, S. 119).

Neben der Zunahme der Femsehdauer bei erweitertem Angebot ist feststellbar: Niedrige soziale Schicht, familiäre Problemsituationen (Arbeitslosigkeit, AlleinErzieher-Situation, Berufstätigkeit beider Eltem) und kommunikative Defizite in der Familie sind mit einseitig erhöhtem und gerade nicht anregendem Femsehgebrauch gekoppelt.

Ein weiterer Aspekt ist in diesem Zusammenhang die Umschichtung der Publika von den öffentlich-rechtlichen Sendem zu den privaten. Wie ECKARDT (1989) zeigte, fallen bei den 6- bis 13jährigen Kindem aus allen Femsehhaushalten fast $64 \%$ der Sehzeiten auf die Nutzung von ARD, ZDF und III. Programme; in den Kabelhaushalten betreffen hingegen $68 \%$ der Nutzungen die privaten Sender. Die Änderung ist in der Kindergruppe deutlicher als in der Erwachsenenpopulation (DARSCHIN \& FRANK, 1991, S. 180). 1994 entfielen von der durchschnittlichen Sehdauer bei Kindem 24 Minuten auf die öffentlich-rechtlichen Sender $(=26 \%)$, aber 68 Minuten $(=74 \%)$ auf die privaten (DARSCHIN \& FRANK, 1995, S. 156). Wegen des höheren Gewalt- und des geringeren Informationsangebotes der Privatanbieter ist damit auch eine Änderung der Sehinhalte verbunden.

\subsection{Inhaltliche Aspekte der Femsehnutzung}

Was sehen Kinder gem und oft? Pauschalierend kann man sagen, daß das Fermsehen vorwiegend zu Unterhaltungs- und deutlich seltener zu Informationsoder gar Bildungszwecken genutzt wird. An der Spitze der Beliebtheit stehen bei 6- bis 13jährigen Kindem (KLINGLER \& GROEBEL, 1994, S. 65) Zeichentrickfilme (43\%), deutlich abgeschlagen rangieren Actionfilme (15\%), Familienserien (13\%) oder Tierfilme (11\%). Etwas andere Rangordnungen erhält man, wenn nach der Nutzungshäufigkeit gefragt wird (a.a.O., S. 67); auch hier stehen die Zeichentrickfilme an erster Stelle $(66 \%)$, gefolgt von Sendungen für Kinder (38\%), lustigen Filmen (37\%), Sendungen mit Tieren (37\%) und Actionfilmen $(34 \%)$. Eine Sonderstellung nimmt die nicht vermeidbare Werbung ein $(48 \%)$. Übereinstimmend schlecht bewertet und entsprechend selten genutzt sind Nachrichtensendungen. Die im Vergleich zur Präferenz (1\%) höhere Nutzung von Nachrichtensendungen (11\%) ist auf das „Mitsehen“ dieser Femsehangebote zurückzuführen und nicht auf eine gezielte Suche nach dieser Programmsparte.

Welche inhaltlichen Präferenzen sind für Jugendliche typisch? Aufgrund von Analysen der GfK-Daten stellten van EIMEREN und KLINGLER (1995, S. 214) fest, daß Jugendliche ein eingeschränktes Nutzerprofil aufweisen. Z.B. besteht bei 
Jugendlichen hinsichtlich der Nutzung des ARD-Programms eine besonders große Kluft zwischen dem Angebot von Information (31\%-Programmanteil) und seine Nutzung (15\% Nutzungsanteil).

Nach GfK-Auswertungen nehmen die Musiksender (mtv, Viva) keine substantiellen Zeitanteile ein, dies selbst bei Jugendlichen (trotz ihrer deutlichen Musikvorlieben) nicht. Das soll aber nicht heißen, es handelte sich hier um zuschauerleere Kanäle. Ohne den Nutzeranteil zu quantifizieren, sind auch einige Befunde zu den Hörem von mtv und VIVA bekannt (FrielingSDORF \& HAAS, 1995): Erwartungsgemäß handelt es sich um besonders musikorientierte Personen, sowohl in Hinblick auf die Nutzung selbst wie auch die Thematisierung von Musik im Freundeskreis. Die Zuwendung zu den Musikkanälen ist zufälliger (man zappt eher in diese Programme, wenn auf den anderen Sendem nichts Interessantes kommt) und kurzfristiger als die zu anderen Kanälen. Die permanente „Musikkulisse" im Hintergrund ist eher selten, obwohl sich eine Tendenz in Richtung Radioersatz zeigt; eine ausschließliche Zuwendung zu einem solchen Sender ist nur bei etwa jedem zweiten Nutzer gegeben.

Noch eine Bemerkung soll zum Thema der Nutzung gewalthaltiger Angebote im Fermsehen gemacht werden. Wie die Inhaltsanalyse der Femsehangebote von GROEBEL und GleiCH (1993) gezeigt hat, sind bedeutende Anteile der Sendezeiten mit Gewalthandlungen gefüllt. Die Chance, bei zufälligem Einschalten auf Gewaltszenen zu treffen, ist wesentlich höher als die Chance, Prosozialität gezeigt zu bekommen. Das Gewaltangebot ist in den Privatkanälen zudem wesentlich größer als bei dem öffentlich-rechtlichen Femsehen. Kinder haben sich aber sehr intensiv den Privatanbieterm zugewandt; dies hat zur Folge, daß der Gewaltfilmkonsum über das Medium Fermsehen angestiegen ist. Man wird aufgrund dieser Entwicklungen nicht fehlgehen, im Femsehen eine Einstiegsdroge für den Gewaltkonsum zu sehen.

\subsection{Die soziale Situation beim Femsehen}

Die überwiegende Fernsehnutzung bei Vorschulkindern findet in der eigenen Familie statt (94\%). Zwei Drittel dieser Zeit ist ein Erwachsener anwesend, die restliche Zeit wird zu gleichen Teilen alleine oder mit Geschwistem femgesehen (TiETZE, 1990, S. 270). Nach der „Kinder und Medien Studie 1990“ (KLINGLER \& Groebel, 1994, S. 146) nimmt die Sehsituation mit Erwachsenen zu, je später femgesehen wird (Gipfel ca. $20 \mathrm{Uhr}$ ), mit anderen Kindem wird häufiger früher gemeinsam geschaut (zwischen ca. 18 und $20 \mathrm{Uhr}$ ). Dies bedeutet, daß bis etwa $20 \mathrm{Uhr}$ die Entscheidung über das Programm entweder alleine getroffen wird oder daß Absprachen mit anderen Kindern nötig sind, nicht aber mit Erwachsenen. Als
Gesprächspartner über das Femsehen stehen Freunde (48\%) an erster Stelle (mit zunehmender Tendenz nach dem Alter), dann kommen die Mütter (37\%) und Väter (20\%); $12 \%$ der Kinder meinen, sie hätten niemand, mit dem sie über das Fernsehen sprechen können (für ältere vgl. LUKESCH et al. 1994, S. 68). Demnach findet zwar die Femsehrezeption überwiegend im familiären Kreis statt, die aktive Aufarbeitung der Inhalte aber eher unter Freunden.

Das elterliche Verhalten scheint von gesicherter Vorbildwirkung für Jugendliche zu sein; in einer Studie von Oswald und KuHN (1994, S. 38) war die Femsehhäufigkeit der Eltem (innerhalb eines multiplen Vorhersagesystems) der beste Prädiktor für die Fernsehdauer von Jugendlichen. Aber auch die Beziehung zu Gleichaltrigen spielt eine große Rolle: Je größer der Freundeskreis, je häufiger am Abend ausgegangen wird und je häufiger man sich bei Kummer an seine Freunde wenden kann, desto seltener sieht man ferm. Hingegen korreliert die eigene Fernsehdauer in gleichsinniger Weise mit der Femsehhäufigkeit des(r) besten Freundes(in).

\subsection{Eltemverhalten gegenüber dem Fermsehen}

Eltem zeichnen von sich generell das Bild, daß sie gegenüber dem Fernsehkonsum ihrer Kinder kritisch eingestellt seien. Von den Eltem 6- bis 16jähriger wollen z.B. auf das Lesen nur $21 \%$ Einfluß nehmen, während dies beim Fernsehen $57 \%$ der Eltern für nötig erachten (KÖCHER, 1988). Über $90 \%$ aller Mütter wie auch Väter sagen z.B., daß ihre Kinder nicht alles anschauen dürfen, was im Fernsehen kommt, oder sie lehnen es ab zuzugeben, die Kinder zur eigenen Entlastung femsehen zu lassen (KLINGLeR \& Groebel, 1994, S. 49f). Ebenso überwiegen Meinungen, daß das Femsehen negative Wirkungen nach sich ziehen könnte (z.B. stimmen jeweils mehr als die Hälfte der Eltem den Statements zu, durch das Fernsehen verkümmere die Phantasie der Kinder, es mache die Kinder nervös, lasse ihnen zu wenig Zeit zum Spielen oder vermittle ihnen ein einseitiges und unvollständiges Bild von der Wirklichkeit). Zwischen dieser plakativ medienkritischen Haltung und den medienerzieherischen Handlungen der Eltem besteht allerdings eine deutliche Kluft.

\section{Fermsehen und die Nutzung anderer Medien}

Spricht man von der Multi-Media-Generation, so ist damit impliziert, daß Kinder und Jugendliche in vielfältiger Weise mit Medien umgehen. Da es im gegebenen Kontext nicht möglich ist, systematisch auf alle anderen Mediennutzungen einzu- 
gehen (LUKESCH, 1996), soll ersatzweise der Zusammenhang zwischen dem Leitmedium Femsehen und der Nutzung von Druckmedien und von Video angedeutet werden. Allgemein ist aber bekannt, daß mit dem Ausmaß des Femsehens die Nutzung von Tele- und Videospielen, der Kinobesuche, das Musikhören, das Anschauen von Videofilmen und Computemutzungen ansteigen (SPANHEL, 1990, S. 158).

\subsection{Femsehen und Lesen}

Lesen ist eine kognitiv aufwendigere und voraussetzungsreichere Tätigkeiten als Fernsehen. Bei Kindem und Jugendlichen sind zudem Probleme in Richtung zu geringer Lesekompetenz nachgewiesen (LEHMANN, 1995). Quantitativ rangiert das Lesen deutlich hinter den Femsehzeiten; die Tagesreichweite für das Lesen beträgt bei Kindem in Deutschland 30\%, die durchschnittliche Lesedauer 20 Minuten (KLINGLeR \& GROEBEL, 1994, S. 59). Das Lesen nimmt zwar von der Vorschulzeit bis zum Ende der Pflichtschulzeit zu. Danach hält dieser Trend nicht an, es kommt zu dem sog. Phänomen des „Leseabbruchs“ (d.h. der Institution Schule gelingt es offensichtlich nicht, eine über die Schulzeit hinaus wirkende Lesemotivation aufzubauen). In der Jugend und Medien Studie 1984 war bei den 12- bis 15jährigen eine 25 minütige Beschäftigung mit Büchem vorhanden, diese reduzierte sich bei den Altersgruppen von 16 bis 24 Jahre auf 17 Minuten (LUKESCH et ed., 1994). Bei den etwa 20jährigen steigt das Lesen insgesamt durch z.T. längere Zeitungslektüre wieder an (BoNFADELLI et al., 1986, S. 143).

Zur Beantwortung der Frage, ob als säkularer Trend ein Rückgang des Lesens auszumachen ist, kann auf Daten von BoNFADELLI (1988) verwiesen werden Während sich die Fernsehreichweiten über einen Zehnjahreszeitraum nur wenig verändert haben, hat der Anteil der Leser pro Tag in allen Altersgruppen deutlich abgenommen. Auch in der als noch sehr „lesefreundlich“ zu qualifizierenden Shell-Studie von 1985 (Jugendwerk der Deutschen Shell, 1985, S. 191) wird im Generationsvergleich 1954 vs. 1984 ein Rückgang der Jugendlichen, die Lesen als ihre liebste Freizeittätigkeit bezeichnen, von 35 auf $30 \%$ festgestellt. In der auf einer Datenerhebung von 1991 beruhenden Studie von LEHMANN (1995) hat sich dieser Prozentsatz weiter verringert (Drittklässler in den neuen Bundesländem, die Lesen als ihre liebste Freizeittätigkeit angeben: $29,7 \%$, in den alten Bundesländem $32,3 \%$; Achtklässler 22,8\% zu 25,3\%).

Ein Zusammenhang zwischen der Entwicklung der Lesefreude und dem Femsehen kann in dem Rückgang des Vorlesens seit den Anfängen des Femsehens gesehen werden: Nach FILIPSON (1978, S. 67) würden es Eltern ihren Kindern zunehmend "erlauben", vor dem Fernseher einzuschlafen, sie ersparten sich dadurch Einschlafrituale und Gute-Nacht-Geschichten. Da aber Vorlesen und gemeinsames Buchanschauen eine zentrale Anregung für späteres Lesen sind, ist damit für weniger Kinder der Weg zum Leser geebnet (Stem-Magazin, 1985, Nr. 42, S. 68: 8- bis 12jährigen „Leseratten“ wurde in der Kindheit doppelt so häufig vorgelesen als den „Lesemuffeln“).

\subsection{Fermsehen und Video}

Video ist ein gutes Beispiel für das Zusammenwirken von technischen Entwicklungen und ihrer alltäglichen Nutzung. In den 70er und noch zu Beginn der 80er Jahre war die Videotechnologie so unhandlich, daß sie keinen Eingang in die Haushalte finden konnte. Erst mit der Erfindung der Videokassette und der Einigung auf einen allgemeinen Standard hat dieses Gerät eine weite Haushaltsverbreitung gefunden. Die gehandelten Verbreitungszahlen über diese Geräte sind aber unterschiedlich, nach der „Kinder und Medien Studie 1990“ (KLINGLER \& GroEBEL, 1996, S. 27) sind in $58 \%$ der west- und in $26 \%$ der osteutschen Haushalte Videorecorder vorhanden, nach der MA '94 sind in $73 \%$ der West- und in $62 \%$ der Osthaushalte Videorecorder verfügbar.

Nach aktuellen Reichweitedaten schauen $8 \%$ der 6- bis 13jährigen Kinder pro Tag Video, wobei die Jungen dies häufiger als Mädchen tun (KLINGLER \& Groebel, 1994, S. 59). Das Anschauen von Videos nimmt bei Kindem im Durchschnitt nicht sehr viel Zeit ein: Nach der ARD/ZDF-Studie sind es täglich 6 Minuten bei den Westkindern (bzw. 8 Minuten bei den Ostkindem). Die Verweildauer beträgt allerdings 82 Minuten (KLINGLER \& GROEBEL, 1994, S. 60), was sehr gut der Länge eine Spielfilmes entspricht. Auch bei Jugendlichen ist die durchschnittliche tägliche Nutzung von Video mit 8 Minuten relativ gering; im Vergleich zum Durchschnitt der deutschen Bevölkerung (3 Minuten) aber deutlich höher (VAN EIMEREN \& KLINGLER, 1995, S. 213).

Sowohl aufgrund der Reichweitenangaben wie auch der Nutzungszeiten sind also die Befürchtungen in bezug auf Video zu relativieren, gäbe es nicht die spezielle Qualität des Videoangebots. Die von Eltem und professionellen Pädagogen artikulierte Sorge in bezug auf Video begründet sich darauf, daß in den über 16000 ausleihbaren Videofilmen ein beträchtlicher Bestand an jugendgefährdenden Produkten enthalten ist (in dem Mitteilungsblatt der BPjS vom Mai 1996 sind 2581 indizierte Videofilme aufgeführt, 135 sind aufgrund ihrer menschenverachtenden Gewaltdarstellungen und ca. 100 wegen sog. harter Pomographie beschlagnahmt). Die Jugendschutzgesetzgebung konnte bislang nicht gewähr- 
leisten, daß Kinder und Jugendliche keinen Zugang zu diesen Filmen erhalten (LuKESCH et al., 1989).

Man kann daher zu der Schlußfolgerung kommen, das Medium Video werde zwar selten genutzt, sei aber der Weg, über den sehr massive Gewaltangebote an Kinder und Jugendliche herankommen. Nach vorliegenden Ergebnissen von 1989 haben von den 12- bis 13jährigen Kindern $19 \%$ indizierte und 5\% beschlagnahmte Filme unter ihren Lieblingsfilmen genannt, in höheren Altersgruppen steigt diese Zahl weiter an, z.B. sind unter den 16jährigen 42,5\% Kenner indizierter und $14,7 \%$ von beschlagnahmten Videos (LUKESCH et al., 1989, S. 74). Betrachtet man spezielle Gruppen Jugendlicher, so sind noch wesentlich höhere Zahlen zu berichten (unter 16jährigen männlichen Berufsschülern sind z.B. 62,7\% Kenner indizierter und $27,1 \%$ beschlagnahmter Videos; SCHEUNGRAB, 1989). In den neuen Bundesländern ist eine noch höhere Nutzungsquote von Gewaltfilmen zu finden nach LUKESCH (1992) waren unter den 13jährigen 47,5\% Nutzer indizierter Filme und 5,1\% Nutzer beschlagnahmter Videofilme.

WEISS (1993) hat herausgearbeitet, daß sich der Gewaltfilmkonsum zusehends in die jüngeren Alterssegmente verlagert: Fast jeder zweite Schüler hat seinen ersten Horror- bzw. Gewaltfilm vor dem 10. Lebensjahr gesehen (Vorschulkinder $5 \%$, Grundschule $42 \%$, Sekundarstufe I $58 \%$ ). Jeder 3. Einstiegsfilm war indiziert und ca. $15 \%$ davon beschlagnahmt. Bedeutsam ist auch der Befund, wonach das Einstiegsalter bei späteren Exzessivsehern wesentlich früher lag als bei Seltensehern von Horror- und Gewaltfilmen. In den letzten Jahren (zwischen 1989 und 1992) sind außerdem weitere Steigerungsraten bezüglich der Nutzung von Gewaltfilmen dokumentiert.

Das Ansehen von Videos ist eine Aktivität, die einen deutlichen Peergruppenbezug unter älteren Kindern bzw. Jugendlichen aufweist. Nach der Jugendmedienstudie (LUKESCH et al., 1994, S. 113) ist die häufigste Sehsituation die mit Freunden (86\%), dann kommen Geschwister/Verwandte $(65 \%)$ und erst danach die Eltern (52\%). Noch deutlicher sind die Unterschiede, wenn nach Aussprachepartnern gefragt wird, hier übertreffen die Freunde (45\%) um ein Mehrfaches die Eltern (14\%) oder die Geschwister (14\%) bzw. andere Personen (7\%). Diese Tendenzen sind nicht außergewöhnlich, sondern entsprechen den allgemeinen Entwicklungsbestrebungen weg von der Familie zugunsten stärkerer Orientierung an Gleichaltrigen.

Video hat das Fernsehen nicht beeinträchtigt, sondern die Bindung an die Fernsehapparate erhöht (DARSCHIN \& FRANK, 1986, S. 221; ähnlich auch SPANHEL, 1990, S. 158), d.h. die Videozeit wird nicht von der Fernsehzeit abgeknapst, sondern ist zur Fernsehzeit hinzuzuzählen: Bei 6- bis 13jährigen Kindern betragen die täglichen Bildschirmaktivitäten bei Vorhandensein eines Videorecorders 158 Mi- nuten, in Haushalten ohne Videorecorder sind es nur 108 Minuten. Diese Unterschiede sind zum einen dem Effekt der Einführung des neuen Haushaltsgerätes, zum anderen einem Selbstselektionseffekt zuzuschreiben. Unter den intensiven Fernsehern sind auch signifikant mehr Videonutzer.

Die Schule ist die Institution, von der alle Kinder und Jugendlichen erreicht werden. Man wird daher nicht fehlgehen, eine systematische Medienerziehung, die über die Orientierung der Schule an Geschriebenem hinausgeht, zu fordern. Dabei bieten sich fünf Bereiche für medienerzieherische Maßnahmen an, und zwar (1) die Erziehung zu reflektierter Mediennutzung unter Abwägung von Handlungsalternativen, (2) Medienanalyse und -kritik, (3) Mediengestaltung, (4) Aufarbeitung von Medienwirkungen i.e.S. (z.B. Auseinandersetzung mit medienbedingten Emotionen, Analyse medienvermittelter Vorstellungen über die Realität) und (5) indirekte Maßnahmen zur Unterstützung der Medienerziehung (z.B. Förderung von Prosozialität oder moralischer Urteilsfähigkeit).

\section{Literatur:}

Aregger, J. \& Steinmann, M.: Kinder als Radio- und Fernsehpublikum. Bern: SRGForschungsdienst 1989.

BESSLER, H. \& ZIMMERMANN-SChÜRINGS, M.: Lebensgewohnheiten von Kindern. Auswertung einer Stichtagsbefragung von Kindern in Fernsehhaushalten. In D. STOLTE (Hrsg.), Das Fernsehen und sein Publikum. Mainz: Hase \& Koehler 1973, (S. 201-253).

BonfaDelLI, H.: Das Leseverhalten von Kindern und Jugendlichen. Leselandschaft Schweiz. Zürich: Schweizerisches Jugendbuch-Institut 1988.

Bonfadelli, H., Darkow, M. Eckhardt, J., Franzmann, B., Kabel, R. Meier, W., Weger, H.-D. \& WIEDEMANN, J.: Jugend und Medien. Eine Studie der ARD/ZDF-Medienkommission und der Bertelsmann Stiftung. Frankfurt a.M.: Metzger 1986.

Darschin, W. \& FranK, B.: Tendenzen im Zuschauerverhalten. Fortsetzungsbericht zur Fernsehnutzung im Jahre 1985. In: Media Perspektiven, 4/86, S. 209-222.

DARSCHIN, W. \& FRANK, B.: Tendenzen im Zuschauerverhalten. Fernsehgewohnheiten und Fernsehreichweiten im Jahr 1990. In: Media Perspektiven, 3/91, S. 178 - 193.

DARSCHIN, W. \& FRANK, B.: Tendenzen im Zuschauerverhalten. Fernsehgewohnheiten und Fernsehreichweiten im Jahr 1994. In: Media Perspektiven, 4/95, S. 154-165.

ECKHARDT, J.: Aktuelle Ergebnisse der GfK zum Thema Fernsehnutzung von Kindern. BadenBaden: SWF-Medienforschung 1989.

van EIMERen, B. \& KlingleR, W.: Elektronische Medien im Tagesablauf von Jugendlichen. Nutzungsdaten 14- bis 19jähriger zu Fernsehen, Video, Hörfunk und Tonträgern. In: Media Perspektiven, 5/95, S. 210-219.

Feierabend, S. \& Windgasse, T.: Was Kinder sehen. Eine Analyse der Fernsehnutzung 1995 von 3- bis 13jährigen. In: Media Perspektiven, 4/96, S. 186-194.

FiLIPSON, L.: Die Rolle des Fernsehens im Leben von Vorschulkindern. In E. HÖMBERG (Bearb.), In:Vorschulkinder und Fernsehen. Empische Untersuchungen in drei Ländern. München: Saur 1978 , S. 63-78 
Firnkes, M., Keilhackek, M. \& Voui, G.: Das Fernsehen im Vorschulalter. Empirische Studie über Funktion und Wirkung des Fernsehens bei Kindern im Vorschulalter. München: Wissenschaftliches Institut für Jugend- und Bildungsfragen in Film und Fernsehen 1973

Frielingsdorf, B. \& HaAs, S.: Femsehen zum Musikhören. Stellenwert und Nutzung von MTV und VIVA beim jungen Publikum in Nordrhein-Westfalen. In: Media Perspektiven, 7/95, S. 331-339

Furu, T.: Function of television for children and adolescents. Tokyo: Sophia University 1971.

Groebel, J. \& Gleich, U.: Gewaltprofil des deutschen Fernsehprogramms. Eine Analyse des Angebots privater und öffentlich-rechtlicher Sender. Opladen: Leske + Budrich 1993.

Hurrelmann, B.: Fernsehen in der Familie. Auswirkungen der Programmerweiterung auf den Mediengebrauch. Weinheim: Juventa 1989.

Infratest: Fernsehen im Alltag von Kindern. In: ARW (Hrsg.), Kinder, Medien, Werbung. Frankfurt a.M.: Metzner Verlag 1981 (Band 1 der Schriftenreihe Media Perspektiven), S. 297-601

Jugendwerk der Deutschen Shell (Hrsg.): Jugendliche und Erwachsene '85. Generationen in Vergleich. Opladen: Leske \& Budrich 1985.

Klingler, W. \& Groebel, J.: Kinder und Medien 1990. Eine Studie der ARD/ZDF-Medienkommission. Baden-Baden: Nomos 1994.

KLINGLER, W. \& Windgasse, T.: Was Kinder sehen. Eine Analyse der Fernsehnutzung von 6- bis 13jährigen. In: Media Perspektiven, 1/94, S. 2-13.

KOCHER, R.: Familie und Lesen. Eine Untersuchung über den Einfluß des Elternhauses auf das Leseverhalten. Archiv für Soziologie und Wirtschaftsfragen des Buchhandels LXIII. Frankfurt a.M 1988.

KкөтZ, F.: Alleinsehen im „Fernsehfluß“*. Rezeptionsmuster aus dem Blickwinkel individueller Fernsehnutzung. In: Media Perspektiven, 10/94, S. 505-516.

LEHMANN, R.: Leseverständnis und Lesegewohnheiten deutscher Schüler und Schülerinnen. Reihe Beltz - Stiftung Lesen. Weinheim: Beltz 1995.

LUKESCH, H.: Aktuelle Videokonsumgewohnheiten bei Kindern und Jugendlichen in den fünf neuen Bundesländern. BPS-Info der Bundesprüfstelle für jugendgefährdende Schriften, I, 1992, 3-5.

LukesCh, H.: Mediennutzen und Mediennutzung bei Kindern. In M. MarkefKa \& B. NaucK (Hrsg.). In: Handbuch der Kindheitsforschung. Neuwied: Luchterhand, (S. 481-489)

LuKESCH, H.: Die Nutzung von Medien durch Kinder und Jugendliche unter quantitativen Aspekten. In Bayerisches Staatsministerium für Unterricht, Kultus, Wissenschaft und Kuns (Hrsg.), Sammelwerk Medienzeit. Donauwörth: Auer 1996 (im Druck).

Lukesch, H., KäGi, H., Karger, G. \& TASChleR-Pollacek, H.: Video im Alltag der Jugend. Quantitative und qualitative Aspekte des Videokonsums, des Videospielens und der Nutzung anderer Medien bei Kindern, Jugendlichen und jungen Erwachsenen. Regensburg: Roderer 1989.

Lukesch, H., Kischkel, K.-H., Amann, A., Birner, S., Hirte, M., Kern, R., Mousburger, R., Müller, L., SChubert, B. \& SChuller, H.: Jugendmedienstudie. Regensburg: Roderer, '1994.

Oswald, H. \& KuHN, H.-P.: Fernsehhäufigkeit von Jugendlichen. Analyse des Einflusses von Eltern und Freunden. In: Media Perspektiven, 1/94, S. 35-41

Rosengren, K.E. \& W INDAhL, S.: Media matter. TV use in childhood and adolescence. Norwood, NewJersey: Ablex Publ. Corp 1989.

SAXer, U., LANGenbUCHER, W. \& Fritz, A.: Kommunikationsverhalten und Medien. Lesen in de modernen Gesellschaft. Gütersloh: Bertelsmann Stiftung 1989.
SCHEUNGRAB, M.: Videokonsum von Jugendlichen und jungen Erwachsenen. Neuere Ergebnisse zur Verbreitung und Nutzung des Mediums Video. In: Empirische Pädagogik, 3/89, S. 257-269.

SchneEwind, K.A., Beckmann, M. \& Engfer, A.: Eltern und Kinder. Stuttgart: Kohlhammer 1983.

SPANHEL, D.: Jugendliche vor dem Bildschirm. Neueste Forschungsergebnisse über die Nutzung der Videofilme, Telespiele und Homecomputer durch Jugendliche. Weinheim: Deutscher Studienverlag ${ }^{2} 1990$.

Der Stern: Ausgelesen. In: Stern-Magazin, 42/85, S. 64-68.

TIETZE, W.: Mediennutzung bei Vorschulkindern: Umfang, Zeiten, sozialer Kontext und Bedingungen. In: Empirische Pädagogik, 4/90, S. 263-288.

WEISS, R.: Gewaltmedienkonsum. Video-Gewalt 1992. Befragung von 12-16 jährigen Schülern aus 6.-9. Klassen im Bezirk des Oberschulamts Stuttgart. Stuttgart: Mimeo 1993

\section{Kurzbiographie:}

Dr. Helmut LukesCh, geb. 1946, nach dem Besuch der Bundeslehrerbildungsanstalt in Salzburg Studium von Psychologie, Pädagogik und Philosophie an den Universitäten Innsbruck und Salzburg; Promotion 1971; Hochschulassistent am Institut für Psychologie der Universität Salzburg bis 1973; Assistent im Fachbereich für Erziehungswissenschaft und wissenschaftlicher Mitarbeiter im Zentrum I für Bildungsforschung der Universität Konstanz; Habilitation 1976; seit 1978 Ordinarius für Psychologie an der Universität Regensburg.

Anschrift: Institut für Psychologie, Universität Regensburg, Universitätsstraße 31, D-93040 Regensburg (privat: Lindenstr. 6, D-93195 Wolfsegg). 